\title{
microRNAs: small regulators of human disease
}

Anandwardhan A. Hardikar, PhD

Editor, microRNAs in Diabetes and Obesity

Associate Professor and Australian Future Fellow (ARC)

NHMRC Clinical Trials Centre,

The University of Sydney, Australia

(C) Versita Sp. z o.o.

NoncodingRNAs (ncRNAs) is a large class of structurally and functionally diverse RNA molecules that do not code for any protein. One of the most significant discoveries in the past 2 decades has been the identification of small regulatory noncoding RNAs; microRNAs (also referred to as "miRNAs" or "miRs"). The discovery of microRNAs and the understanding of regulatory mechanisms that they offer in "fine tuning" the adaptation and presentation of individual genomes is overwhelming.

Originally described in animals by Victor Ambros [1] and Gary Ruvkin [2] in 1993, microRNAs have now been recognized to impact human health in numerous ways. Discovery of such regulatory molecules have assigned a critical role to the originally thought "junk DNA". Bacteria and other prokaryotic organisms have the least amount of non-coding ("junk") DNA as compared to the more evolved species. This "junk DNA" was believed to have accumulated over several centuries of evolution as a result of evolutionary modifications, transposons as well as parasite and viral infections. Following completion of the human genome project in 2001 , it was revealed that only up to $2 \%$ of our genome codes for all the proteins that make up our bodies. This was very interesting as humans, having developed complex physiologies, were believed to carry many more protein-coding genes. Instead, it was discovered that insects have twice as many protein-coding genes $(\sim 13,500)$ than yeast $(\sim 6,000)$ and that increase in cell number or complexity is not related to number of protein-coding genes. There are 19,000 protein-coding genes in C. elegans, for its 959 -cell body plan as compared to similar number $(\sim 22,000)$ of protein-coding genes in humans for $\sim 10$ trillion cells. One major difference between slugs and humans is the relative proportion of non-coding DNA. It is now demonstrated that ncRNAs can influence the expression of over $30 \%$ of proteincoding genes and play important homeostatic roles to regulate gene expression during development and disease.

Today, it is well known that microRNAs play an important role in normal development of endocrine pancreas and may also regulate progression of diabetes and its complications. The number of publications related to microRNAs and Diabetes have increased significantly over the last 10 years (Figure 1A).
The importance of these publications in understanding other biologies has also greatly increased based on a high citation rate of these articles (Figure 1A). A number of grant applications and

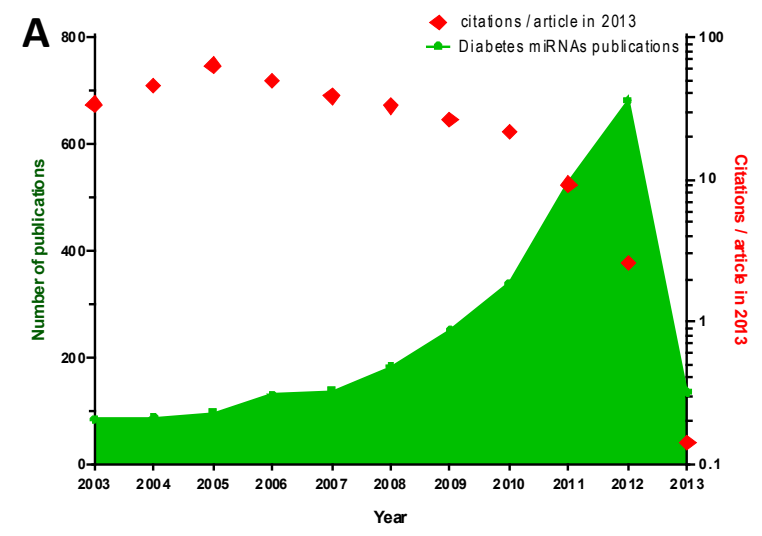

B

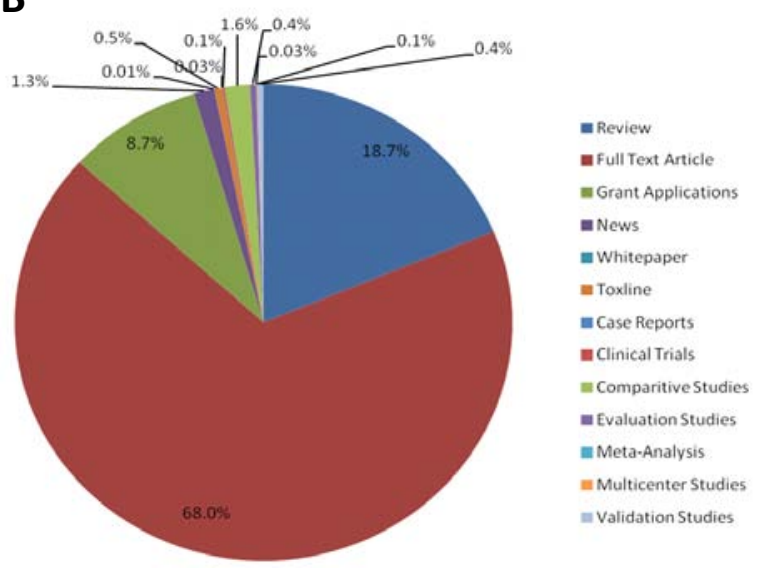

Figure 1. Scientific interest in microRNAs

A) Number of publications in the last 10 years that discuss the role of microRNAs with relation to Diabetes along with the number of citations received by each of these articles as identified in 2013. Research articles were identified using appropriate search terms through Scopus. B) A distribution of microRNA studies worldwide. Numbers represent $\%$ of total hits obtained through Quertle database (http://www.Quertle. info/) for the appropriate search terms (Analysis / compilation by Sarang Satoor and Ryan Farr). 
clinical trials are now seen to be focussed on understanding the role of microRNAs (Figure 1B) in various diseases.

Finally, after 20 years from their discovery, I welcome you all to this new journal that will publish key findings from research related to microRNAs in diabetes and obesity. This new journal provides you with an elegant platform to publish a vast range of articles, from short communications and pictures in small RNA world to larger full length research publications and extensive reviews. The philosophy behind our publication is that every piece of data is important to solving a bigger puzzle and we therefore offer various publication types to present your work. We also welcome commentaries on articles published elsewhere but relating to the central theme of this journal. One of the other unique features of the journal is immediate publication following editorial decision. Versita offers a unique platform wherein all

\section{References}

[1] Lee, R. C.; Feinbaum, R. L.; Ambros, V. (1993). "The C. Elegans heterochronic gene lin-4 encodes small RNAs with antisense complementarity to lin-14". Cell 75 (5): 843-854. articles will be immediately published online rather than waiting to be assigned and printed in periodic issues. The editorial board comprises of some of the most eminent researchers in the area of microRNA, obesity, diabetes and its complications. We are backed by one of the most experienced publishers, who publish 400 journals in over 24 different fields. I take this opportunity to thank you all who have sent mails from different parts of the world, expressing enthusiasm for this new journal. Special thanks to all our Assistant Editors and Language Editors for their support and specifically to Agnieszka Ludwig-Galezowska, Ewa Zurawska-Seta and all the Editorial team at Versita for their time and assistance in making this possible.

I look forward to welcoming you all to this new platform for publishing your exciting discoveries on microRNAs in Diabetes and Obesity.

[2] Wightman, B.; Ha, I.; Ruvkun, G. (1993). "Posttranscriptional regulation of the heterochronic gene lin-14 by lin- 4 mediates temporal pattern formation in C. Elegans". Cell 75 (5): 855862. 